\title{
Holey Fibres: Properties, Applications and Future Directions
}

\author{
Tanya M. Monro, Joanne C. Baggett, Walter Belardi, \\ Kentaro Furusawa, J.H.V. Price and D.J. Richardson \\ Optoelectronics Research Centre, University of Southampton, SO171BJ, UK
}

\section{ABSTRACT}

Holey optical fibres offer a much wider range of optical properties than is possible in conventional optical fibres. These properties include single-mode guidance at all wavelengths, novel dispersion properties including broadband dispersion flattening and anomalous dispersion at visible wavelengths, mode size tailoring over three orders of magnitude, and many more. Recent progress in this rapidly emerging technology will be reviewed, ranging from modelling and fabrication through to devices and applications.

Keywords: microstructured fibres, holey fibres, nonlinear devices, high power delivery, new laser wavelengths

\section{INTRODUCTION}

The area of microstructured optical fibres has developed into a topic of acute fundamental scientific and technological interest in recent years. The growth and development of the field has been explosive since the first demonstration of guidance within such structures in 1996, by Phillip Russell and co-workers then at Southampton University [1]. There are now numerous university and industrial laboratories engaged in developing holey fibre technology and seeking to apply it to applications as diverse as dispersion compensation through to metrology. The reason for this frenetic activity is quite simple - holey fibre technology provides unique and technologically enabling optical properties that simply cannot be achieved with conventional fabrication approaches.

Microstructured optical fibres can be classified into three main types. Holey fibres [HFs] possess a solid core surrounded by a cladding region defined by a fine array of air holes that extend along the fibre length ( see Fig.1 ). Holey fibres guide light due to the effective refractive index difference between core and cladding regions, and can be made from a single material, typically silica. The second category relates to those fibres that guide by photonic band gap (PBG) effects and which are referred to as photonic band gap fibres. The third category relates to fibres that guide light through some more ' conventional mechanism, but take advantage of the transverse microstructuring in some other way, for example by the way that the arrangement of holes afifiects the cladding mode distribution. For the purpose of this paper, we concentrate on the fiirst category: the holey fiibre.that arrangement ofi holes afifiects the cladding mode distribution.
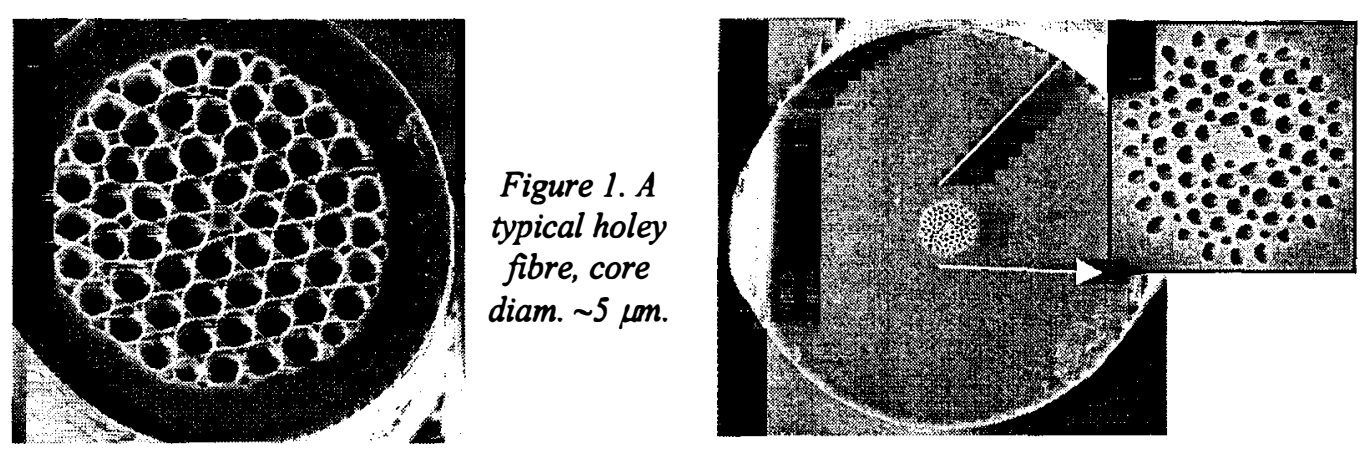

Figure 2.

Figure 1. A typical holey fibre, core diam. $\sim 5 \mu \mathrm{m}$.

\section{BACKGROUND}

\subsection{Fabrication}

Holey fibres are typically fabricated by stacking an array of capillaries in a hexagonal configuration around a solid rod, which ultimately forms the core. The resulting preform is then reduced to fibre dimensions using a conventional fibre drawing tower. By controlling the conditions under which the fibre is drawn, a range of fibre profiles can be produced from a single preform. If a large scale-reduction factor is required, as for the applications described in Section 4, then the microstructured region may be over-clad with a solid jacket. This allows extremely small structure dimensions to be achieved without compromising the practicality and handling of the fibre (see for example Fig.2).

Although the stacking procedure described above typically produces near-periodic refiractive index profiiles, it should be appreciated that. periodicity is not required. Indeed, fiibres with randomly distributed air holes can guide light [2]. 


\subsection{Modelling}

The first method developed to model holey fibres was the effective index model [3], which approximates $\mathrm{HF}$ using an equivalent step-index fibre in the scalar approximation. Although this reduced model can provide some insight into HF operation, it cannot accurately predict modal properties such as the dispersion, which depend critically on the cladding configuration $[4,5,6]$. Note that when the air filling fraction is large and/or the structure scale is small, it is necessary to use a full vector method.

One quite general vector approach to describing the complex spatial index distribution in a HF involves decomposing the refractive index profile and modal fields into plane waves $[7,8]$. However, such techniques are computationally intensive, since they do not take advantage of the localization of the guided modes in the fibre core.

A multipole method has recently been developed to study HFs [9]. This method can be applied to idealized single-material fibres with circular holes, and is well suited to evaluating the confinement losses and polarization properties of $\mathrm{HF}$ designs.

Finally, a hybrid approach, which is efficient, since it uses localized functions to describe the (localized) guided modes, and accurate, because it uses plane waves to describe the index profile, is described in Refs $[4,5,6,10]$. This approach is ideal for predicting the properties of both idealized and actual HFs.

\section{OPTICAL PROPERTIES}

Holey fibres guide light by virtue of a modified variant of the total internal reflection mechanism employed in conventional optical fibres. The basic physical requirement to achieve this form of guidance is that the volume average index in the core region of the fibre is greater than that of the surrounding regions. Note that the hole diameter (d) and hole-to-hole spacing or pitch $(\Lambda)$ of a holey fibre are typically on the scale of the wavelength of light $\lambda$ guided within the fibre. The fundamental physical difference between these fibres and conventional types arises from the way the guided optical mode experiences the cladding region. In a conventional fibre, this is to first-order largely independent of wavelength. In a holey fibre, the large index contrast between glass and air and the small structure dimensions combine to make the cladding index seen by the guided light a strong function of wavelength. For example, short wavelengths can remain tightly confined to the core, and so the effective cladding index is only slightly lower than the core index. However, at longer wavelengths, the mode must sample more of the cladding region, and so the effective index contrast is larger. This unusual wavelength dependence leads to a host of highly unusual and tailorable optical properties, as described below.

Perhaps the most striking property of this fibre type is the fact that fibres with a low air fill fraction $(\mathrm{d} / \Lambda<0.4)$ can be single-moded at all wavelengths [3]. This property has great practical significance, particularly for broadband or short wavelength applications.

Tailoring the scale of the features in the cladding allows the effective mode area of a holey fibre at $1.55 \mu \mathrm{m}$ to be varied over three orders of magnitude from approx. $1 \mu \mathrm{m}^{2}$ (small-pitch, large air filling fraction) to $>1$ $000 \mu \mathrm{m}^{2}$ (large-pitch, small air filling fraction) [4,5]. At the smaller end of this range, it is clear that holey fibres offer a new route towards efficient nonlinear devices, and this is discussed in Section 4. At the other extreme, large mode area holey fibres offer a number of potential advantages over conventional technology (see Section 5).

By combining asymmetry with the large index contrast and small core dimensions, it is possible to produce highly birefringent HF [11]. An example is shown in Fig.3: in this case, the small elliptical core results in enormous form birefringence. Our measured beat length at $1.55 \mu \mathrm{m}$ was $0.3 \mathrm{~mm}$, which is to our knowledge the shortest beat length reported in an optical fibre. Small-core HFs are typically polarization-maintaining.

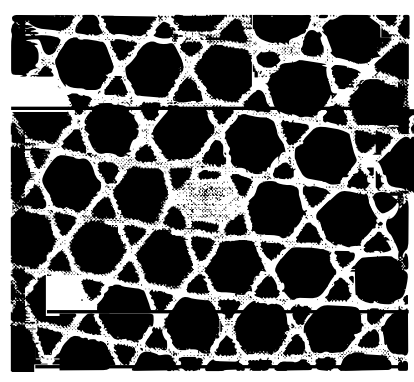

Figure 3.

Polarizationmaintaining holey fibre with an $\mathrm{Yb}^{3+}$ doped central core of dimensions $2.6 \times 1.5 \mu \mathrm{m}$. 
Holey fibres can also exhibit a range of unusual dispersion properties. For example, holey fibre with a small pitch $(\Lambda \sim 1-2 \mu \mathrm{m})$ and large air holes can exhibit anomalous dispersion at short wavelengths $[4,5,6]$. This has made the generation and propagation of optical solitons in the near-IR and visible regions of the spectrum a reality [12].

It is also possible to design HFs with extremely high values of anomalous or normal dispersion $[4,5,13]$, the latter type being of relevance for dispersion compensation applications at $1550 \mathrm{~nm}$. Normal dispersion values as high as $-2000 \mathrm{ps} / \mathrm{nm} / \mathrm{km}$ have been predicted. Other work has shown that broadband dispersion-flattened holey fibres can also be designed $[14,15]$. Such fibres have applications for broadband WDM devices.

Finally, it is worth adding that the holes in holey fibres can be exploited for applications in which an interaction with an evanescent field is required e.g. Raman scattering within a gas, or gas sensing. In order to obtain significantly high overlap between the guided mode and the holes, it is necessary to use fibres with a relatively large air fill fraction $(d / \Lambda>0.6)$ and small structure scale $(\Lambda<\lambda)[16]$.

\section{HIGH NONLINEARITY APPLICATIONS}

Holey fibres with small-scale features (i.e. small $\Lambda$ ) and a large air-filling fraction (i.e. large d/ $\Lambda$ ) can confine the guided mode tightly within the core, resulting in extremely small mode areas, and Figs 2 and 3 show small-core HFs. In such a fibre, modest light intensities can induce significant nonlinear effects. Here a few applications of this high effective nonlinearity are presented briefly.

We have recently demonstrated a $2 \mathrm{R}$ data regeneration device based on a highly nonlinear holey fibre, with an effective mode area of just $2.8 \mu \mathrm{m}^{2}$ at $1.55 \mu \mathrm{m}$ [17]. The $2 \mathrm{R}$ regenerative operation was obtained by combining self-phase modulation (SPM) and offset spectral filtering. Kilometers of conventional fibres are required to generate sufficient SPM for this regeneration process. In this case, just $3.3 \mathrm{~m}$ of HF was needed.

As described in Section 3, small-core HFs can have anomalous dispersion down to visible wavelengths, which raises the possibility of solitons at wavelengths would accessible using conventional fibres. Using the small-scale Ytterbium-doped HF shown in Fig.3, we have demonstrated the operation of a low threshold, tunable, mode-locked soliton laser [18]. With the same fibre, we recently demonstrated a continuously tunable soliton source in the wavelength range 1.06-1.33 $\mu \mathrm{m}$ [19], as shown in Fig.4. This wavelength range is difficult to access using conventional solid state laser technology, and the high effective nonlinearity provided by the $\mathrm{HF}$ allows this source to operate at $\mathrm{mW}$ average input power levels in a $4.7 \mathrm{~m}$ length of fibre.

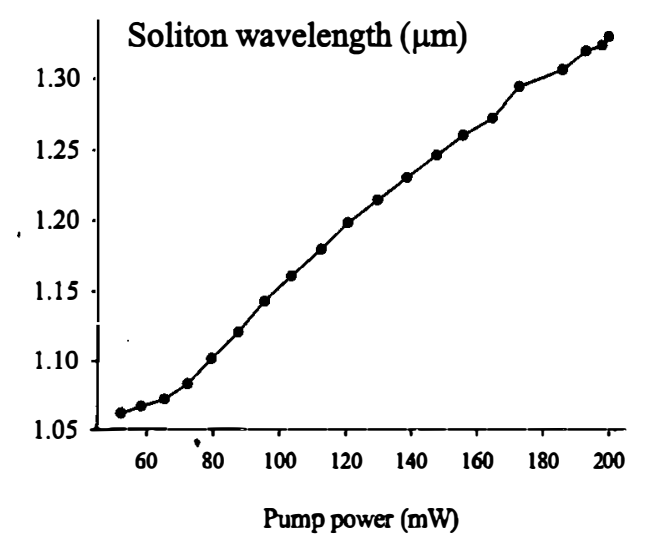

Fig. 4. Tuning curve of soliton wavelength vs. amplifier purno.

Moreover, such a shift in zero-dispersion wavelength to wavelength regions in which there are convenient short pulse sources, coupled to the small associated mode area, also allows the development of efficient supercontinuum sources whose spectral content extends from the out to beyond $1.8 \mu \mathrm{m}$ [20]. Such sources are attractive for many applications including optical sensor systems, pulse compression and the definition of precise frequency standards. 


\section{LARGE MODE AREA FIBRES}

Optical fibers with large mode areas are necessary for applications requiring high power delivery including laser welding and machining, and fiber lasers and amplifiers. Recently, holey fiber technology has emerged as a flexible altemative route towards large mode areas [21].

The largest mode size that can be tolerated in practice is determined by macroscopic bending losses. Recent work shows that holey fibers typically possess comparable bend losses at $1.55 \mu \mathrm{m}$ to similarly sized conventional fibers [22]. Although holey and conventional fibers can exhibit similar mode areas and number of modes at any given wavelength, holey fibers have a distinct advantage for broadband applications due to their ability to be single or few-moded over a large wavelength range. In addition, holey fibers may simplify the fabrication of single-mode fibers with large mode areas at short wavelengths.

\section{OTHER RECENT RESULTS}

It has recently been shown that gratings can be written into holey fibres with a germanium-doped core region [23]. Long-period fibre gratings (LPGs) written in these fibres were shown to be highly insensitive to the external environmental conditions relative to conventional LPGs.

Recently experiments verify that holey fibers can be thermally poled [24]. The flexible range of optical properties possible in a holey fibre should allow significant improvements to be made to the second harmonic efficiency that can be achieved in an optical fibre.

The vast majority of the holey fibres fabricated to date have been made of silica. Recently, we have shown that HFs can be made using compound glasses [25]. These materials offer a range of desirable optical properties such as two orders of magnitude increase in material nonlinearity, and transparency for wavelengths as long as $10 \mu \mathrm{m}$.

\section{CONCLUSIONS}

The novel optical properties possible in holey fibres are beginning to have a significant impact on many areas. Fabrication of holey fibres has now reached the point that $\mathrm{km}$-scale lengths of polymer-coated fibre with losses as low as $3.2 \mathrm{~dB} / \mathrm{km}$ at $1.5 \mu \mathrm{m}$ are possible [26]. Furthermore, HFs fibres can be spliced to conventional fibres [27], which allows ready integration with existing components and systems. In conclusion, holey fibres are a robust and practical new fibre type that has the potential to enable a host of new optical devices for telecommunications and beyond.

\section{ACKNOWLEDGEMENTS}

T.M. Monro and D.J. Richardson acknowledge the support of Royal Society University Research Fellowships. 


\section{REFERENCES}

[1] J.C. Knight et al: All-silica single-mode optical fiber with photonic crystal cladding, Opt. Lett., vol. 21. pp. 1547-1549, Oct. 1996.

[2] T.M. Monro et al: Holey fibers with random cladding distributions, Opt. Lett., vol.25. pp. 206-208, Feb. 2000.

[3] T.A. Birks, J.C. Knight and P.St.J. Russell: Endlessly single-mode photonic crystal fiber, Opt. Lett., vol. 22. pp. 961-963, Jul. 1997.

[4] T.M. Monro et al: Holey optical fibers: An efficient modal model, IEEE J. Lightwave Technol., vol. 17. pp. 1093-1102, Jun. 1999.

[5] T.M. Monro et al: Modeling large air fraction holey optical fibers, IEEE J. Lightwave Technology, vol. 18. pp. 50-56, Jan. 2000.

[6] D. Mogilevtsev, T.A. Birks, P.St.J. Russell: Group-velocity dispersion in photonic crystal fibers, Opt. Lett., vol. 23. pp. 1662-1664, Nov. 1998.

[7] E. Silvestre, M.V. Andrès and P. Andrès: Biorthonormal-basis method for the vector description of opticalfiber modes, J. Lightwave Technol., vol. 16. pp. 923-928, May. 1998.

[8] J.Broeng et al: Analysis of air-guiding photonic bandgap fibers, Opt. Lett., vol. 25. pp. 96-98, Jan. 2000.

[9] T.P. White, R.C. McPhedran, C. Martijn de Sterke: Multipole method for efficient microstructured optical fiber calculations, paper JTuC6, Proc. CLEO'2001, 2001.

[10] T.M. Monro, N.G.R. Broderick and D.J. Richardson, Exploring the Optical Properties of Holey Fibres, in Nanoscale Linear and Nonlinear Optics, M. Bertolotti, Ed., New York: American Institute of Physics, 2000.

[11] A.Ortigosa-Blanch et al: Highly birefringent photonic crystal fibers, Opt.Lett., vol. 25. pp. 1325-1327, Sept. 2000.

[12] W.J. Wadsworth et al: Soliton effects in photonic crystal fibres at $850 \mathrm{~nm}$, Electron. Lett., vol. 36. pp. 5355, Jan. 2000.

[13] T.A. Birks et al: Dispersion compensation using single-material fibers, IEEE Photon. Technol. Lett., vol. 11. pp. 674-677, Jun. 1999.

[14] T.M. Monro et al: Efficient modeling of holey optical fibers, paper FC3, Proc. OFC'99, 1999.

[15] A. Ferrando et al: Designing a photonic crystal fibre with flattened chromatic dispersion, Electron. Lett., vol. 35. pp. 325-327, Feb. 1999.

[16] T.M. Monro, D.J. Richardson and P.J. Bennett: Developing holey fibres for evanescent field devices, Electron. Lett., vol. 35. pp. 1188-1189, Jul. 1999.

[17] P. Petropoulos, T. M. Monro, W. Belardi, K. Furusawa, J.H. Lee and D.J. Richardson: A highly nonlinear holey fiber and its application in a regenerative optical switch, paper TuC3, Proc. OFC'2001, 2001.

[18] K. Furusawa, T.M. Monro, P. Petropoulos and D.J. Richardson, Modelocked laser based on ytterbium - doped holey fibre', Electron. Lett., vol. 37. pp. 560-561, Apr. 2001.

[19] J.H.V. Price, K. Furasawa, T.M. Monro, L. Lefort and D.J. Richardson: A tuneable, femtosecond pulse source operating in the range 1.06-1.33 microns based on an $\mathrm{Yb}$ doped holey fiber amplifier, paper CPD1, Proc. CLEO'2001, 2001.

[20] J.K. Ranka et al.: Visible continuum generation in air-silica microstructure optical fibers with anomalous dispersion at $800 \mathrm{~nm}$, Opt. Lett., vol.25. pp. 25-27, Jan. 2000.

[21] J.C. Knight, T.A. Birks, R.F. Cregan, P.St.J. Russell and J.P. de Sandro: Large mode area photonic crystal fibre, Electron. Lett., vol. 34. pp. 1346-1347, Jun., 1998.

[22] T.M. Monro et al: Comparative study of bend loss in large mode holey and conventional fibres, paper CtuAAl, Proc. CLEO'2001, 2001.

[23] B.J. Eggleton et al: Grating resonances in air-silica microstructured optical fibres, Opt. Lett. vol. 24. pp. 1460-1462, Nov. 1999.

[24] D. Faccio et al: Demonstration of thermal poling in holey fibres, Electron. Lett., vol. 37, pp. 107-108, Jan. 2001.

[25] T.M. Monro et al: Chalcogenide holey fibres, Electron. Lett., vol. 36, pp. 1998-2000, Nov. 2000.

[26] H. Kubota et al: Low-loss, 2km-long photonic crystal fiber with zero GVD in the near IR suitable for picosecond pulse propagation at the 800nm band, CPD3, Proc. CLEO'2001, 2001.

[27] P.J. Bennett, T.M. Monro, and D.J. Richardson: Towards practical holey fibre technology: Fabrication, Splicing, Modelling and Characterization, Opt. Lett., vol. 24. pp. 1203-1205, Sept. 1999. 